\title{
Measuring oblique incidence sound absorption using a local plane wave assumption
}

\author{
E.R. Kuipers, Y.H. Wijnant, and A. de Boer \\ Research Chair of Structural Dynamics and Acoustics \\ Faculty of Engineering Technology - University of Twente \\ P.O. Box 217, NL-7500 AE, Enschede, The Netherlands. \\ \{e.r.kuipers,y.h.wijnant,a.deboer\}@ctw.utwente.nl
}

\begin{abstract}
Summary
In this paper a method for the measurement of the oblique incidence sound absorption coefficient is presented. It is based on a local field assumption, in which the acoustic field is locally approximated by one incident- and one specularly reflected plane wave. The amplitudes of these waves can be determined with an unidirectional sound intensity probe. The local active- and incident acoustic intensity are straightforwardly obtained. The area-averaged sound absorption coefficient is calculated after spatial integration of these quantities over the surface area of interest. Alternatively, one may use a three-dimensional intensity probe. In that case, the determination of the amplitudes of the plane waves can be formulated as a least-squares problem. Measurements performed for a sound absorbing foam demonstrate that accurate results can be obtained, even under non-ideal acoustic conditions. Measurements carried out for a periodic absorber show that the method is accurate below the cuton frequency of scattering as long as the amplitude of the evanescent surface waves is significantly smaller than that of the specularly reflected wave.
\end{abstract}

PACS no. 43.20El, 43.55Ev, 43.55Dt

\section{Introduction}

The availability of sound absorption data for oblique incidence would help in increasing the quality of room acoustic simulations, as shown by Vorländer [1]. However, such data is hardly available; typically one can only obtain the diffuse incidence sound absorption coefficient determined with the reverberation room method acc. ISO-354 [2].

Multiple methods $[3,4,5,6,7,8,9,10]$ can be applied to determine the oblique incidence sound absorption coefficient. These methods mostly determine the specific acoustic surface impedance or the complex sound pressure reflection coefficient, using a model that typically describes the overall acoustic field in front of a planar surface of infinite extent in a semi-free field. In addition, plane- or spherical wave incidence, and a local- or extended reaction surface is assumed. For overviews of acoustic field models the reader is referred to two recent works, by Liu and $\mathrm{Li}$ [11] and Li and Liu [12].

Received 000000 accepted 000000 .
As a result of using an overall model, the directivity characteristics of the source have to be known, large sample sizes are required, and room reflections have to be absent. Whereas the latter requirement can be reasonably fulfilled by measuring in a semianechoic room or by employing a time-windowing technique, it is difficult to obtain pure plane- or spherical wave incidence. Furthermore, the finite dimensions of the sample put a limit on the lowest frequency at which results can be obtained, and the sample's edges give rise to the presence of diffracted waves. Finally, a used local- or extended reaction assumption may not be valid. Therefore, a high chance exists that the actual acoustic field deviates from the ideal field represented by the model, and the obtained acoustic surface impedance or sound absorption coefficient may be inaccurate to a greater or lesser extent.

A possible strategy to reduce the susceptibility of a measurement method to the aforementioned aspects, is to apply area-averaging. In an earlier paper [13], we numerically showed that this approach is effective in reducing the influence of room reflections. It is in contrast to currently available methods which rely on measurements using a spatially fixed set-up, being more susceptible to local deviations in the 
acoustic field that may be caused by edge-diffracted waves or room reflections. Practical use of areaaveraging for measurements in a non-ideal acoustic field has firstly been reported by the authors [14]. In that paper, the LPW-method (Local Plane Wave method) [15, 16, 17, 13] was used. This method is suitable for normal and near-normal incidence.

To also be able to perform area-averaged oblique incidence measurements in non-ideal acoustic fields, we have developed a novel method. This method is the substance of this paper. It is based on a local field assumption, and is referred to as the LSPW (Local Specular Plane Wave) assumption. Accordingly, the novel method is called the LSPW-method. The use of the LSPW assumption allows to determine the oblique incidence sound absorption coefficient without using an overall field model.

This paper is structured as follows: First, the theory will be presented in Sec. 2. Details about the specific implementation for a three-dimensional sound intensity probe, as used by the authors, are given in Sec. 3. In Sec. 4, measurement results for an extended reaction sound absorbing foam and a periodic absorber will be presented. Finally, conclusions and recommendations for further work are given in Sec. 5.

\section{Theory of the LSPW-method}

In the following, we assume that the acoustic field satisfies the Helmholtz equation. The $e^{i \omega t}$-convention is used, i.e. $p(t)=\operatorname{Re}\left[P(\omega) e^{i \omega t}\right]$, vector quantities are bold-faced, and the explicit dependence of $\omega$ is omitted for quantities in the frequency domain.

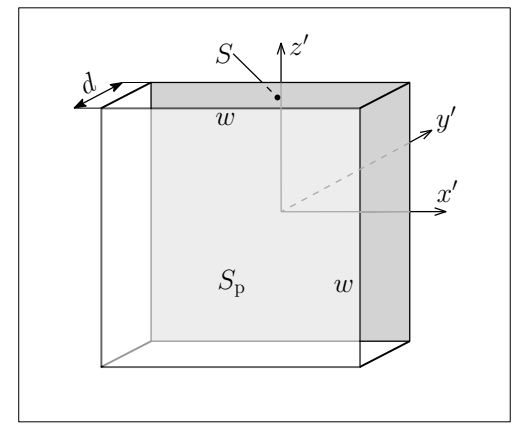

Figure 1. A square, planar material surface $S$ (dark gray), with the measurement surface $S_{\mathrm{p}}$ (light gray) at a distance $d$. the $y^{\prime}$-direction points normally into the material surface.

The theory is presented based on the configuration shown in Fig. 1. We want to obtain an accurate estimate for the oblique incident sound absorption coefficient of the material surface $S$. To this purpose, we define a measurement surface $S_{\mathrm{p}}$, at a small distance $d$ from the material surface $S$.

The effective, area-averaged, oblique incidence sound absorption coefficient for the surface $S_{\mathrm{p}}$ is defined as the ratio of the active (absorbed) acoustic power and the incident power:

$$
\alpha=\frac{W_{\mathrm{ac}}}{W_{\mathrm{in}}}
$$

where $W_{\text {ac }}$ and $W_{\text {in }}$ are the active- and incident acoustic power, respectively. $\alpha$ is an effective sound absorption coefficient as the angle of incidence typically varies over the measurement surface when a spatially fixed source is used. To obtain the area-averaged oblique incidence sound absorption coefficient $\alpha(\psi)$ for a well-defined angle of incidence $\psi$, one must either realize plane wave incidence over the whole surface area of $S_{\mathrm{p}}$, or determine the active- and incident acoustic power associated with a sub-area of $S_{\mathrm{p}}$, at which the angle of incidence lies within small tolerances of the desired angle of incidence.

In the following, it is assumed that the sound source is spatially fixed, and that the source distance and the dimensions of $S_{\mathrm{p}}$ are chosen such that the angle of incidence $\psi$ only varies minimally over the surface $S_{\mathrm{p}}$ of interest, and we define $\tilde{\psi}$ to be the spatially averaged angle of incidence. Then, the active- and incident acoustic power for angle of incidence $\psi$ are obtained by spatial integration of the corresponding intensities over surface $S_{\mathrm{p}}$ :

$$
\begin{aligned}
& W_{\mathrm{ac}}(\tilde{\psi})=\int_{S_{\mathrm{p}}} I_{\mathrm{ac}}(\psi) \mathrm{d} S \\
& W_{\mathrm{in}}(\tilde{\psi})=\int_{S_{\mathrm{p}}} I_{\mathrm{in}}(\psi) \mathrm{d} S
\end{aligned}
$$

where $I_{\mathrm{ac}}(\tilde{\psi})$ and $I_{\mathrm{in}}(\tilde{\psi})$ are active- and incident acoustic intensity in direction $\mathbf{n}$. The local angle of incidence $\psi$ is a function of the spatial position, defined by a spatial vector $\mathbf{r}$ in an overall coordinate system, of the measurement point: $\psi=\psi(\mathbf{r})$. The measurement of the active acoustic intensity can be performed using an unidirectional sound intensity probe, typically being either a pu-probe [18], or a pp-probe [19].

To determine the incident acoustic intensity, one could perform a prior, separate, measurement in a free field. However, such an approach demands for a stable power output of the source, and requires that this measurement is performed at the same position relative to the sound source. To avoid this additional effort, one must determine the active- and 
incident acoustic intensity from a single measurement. Whereas the measurement methods referred to in the introductory section of this paper employ an overall field model, we propose to use a local field assumption.

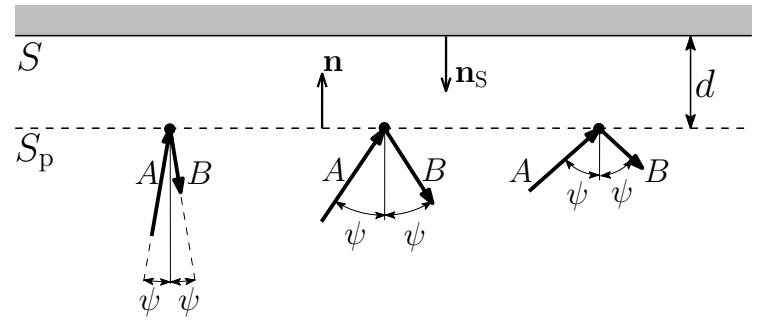

Figure 2. A planar material surface $S$, with the measurement surface $S_{\mathrm{p}}$ at a distance $d . \mathbf{n}_{\mathrm{S}}$ is the surface normal of $S$, and $\mathbf{n}$ is the surface normal vector of $S_{\mathrm{p}}$ (directed towards $S$ ). In each point upon $S_{\mathrm{p}}$, the acoustic field is locally approximated with two waves with complex amplitudes $A$ and $B$, where $\psi$ is equal to the local angle of incidence.

This assumption is illustrated in Fig. 2. In each point upon $S_{\mathrm{p}}$, the acoustic field is locally approximated by an incident plane wave with complex amplitude $A$, and a specularly reflected plane wave with complex amplitude $B$, as shown in Fig. 2. Variations of the angle of incidence, and of the complex amplitudes with space are explicitly allowed.

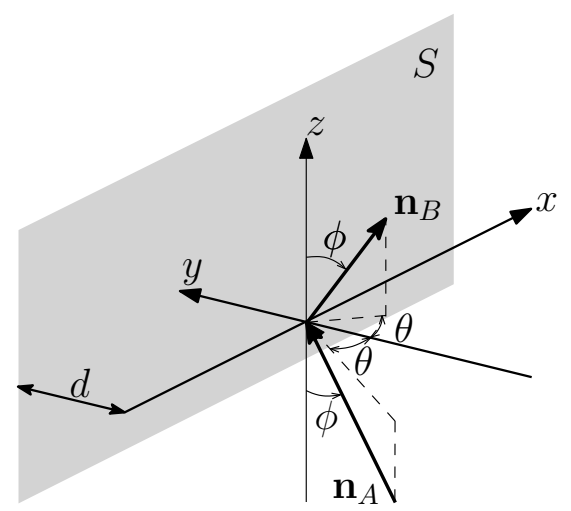

Figure 3. Convention of the propagation direction vectors of $A$ and $B$.

For three dimensions, the direction convention is shown in Fig. 3. It follows that the angle of incidence of the incident wave propagating in direction $\mathbf{n}_{\mathrm{A}}$ with respect to the outward surface normal $\mathbf{n}_{\mathrm{S}}$ (See Fig. 4) equals:

$$
\psi=\arccos (\sin \phi \cos \theta),
$$

so that the propagation directions $\mathbf{n}_{\mathrm{A}}$ and $\mathbf{n}_{\mathrm{B}}$ are separated by an angle $2 \psi$, see Fig. 4 . If, at the measurement point defined by a spatial vector $\mathbf{r}$, the angle of incidence $\psi(\mathbf{r})$ is known, one can determine $A$ and $B$ by using a unidirectional pu- or pp-probe. For a puprobe, we demand that, in every spatial point upon $S_{\mathrm{p}}$, the approximated field, represented by $A(\mathbf{r})$ and $B(\mathbf{r})$, yields the same acoustic pressure and particle velocity as in the actual field. Hence, we obtain

$$
\left[\begin{array}{cc}
1 & 1 \\
\frac{\cos (\psi)}{Z_{0}} & \frac{-\cos (\psi)}{Z_{0}}
\end{array}\right]\left[\begin{array}{l}
A(\mathbf{r}) \\
B(\mathbf{r})
\end{array}\right]=\left[\begin{array}{c}
P(\mathbf{r}) \\
U_{\mathbf{n}}(\mathbf{r})
\end{array}\right]
$$

where $Z_{0}=\rho_{0} c_{0}$ is the specific characteristic acoustic impedance of air. $A(\mathbf{r})$ and $B(\mathbf{r})$ become:

$$
\begin{aligned}
& A(\mathbf{r})=\frac{1}{2}\left[P(\mathbf{r})+\frac{U_{n}(\mathbf{r})}{\cos \psi(\mathbf{r})}\right], \\
& B(\mathbf{r})=\frac{1}{2}\left[P(\mathbf{r})-\frac{U_{n}(\mathbf{r})}{\cos \psi(\mathbf{r})}\right] .
\end{aligned}
$$

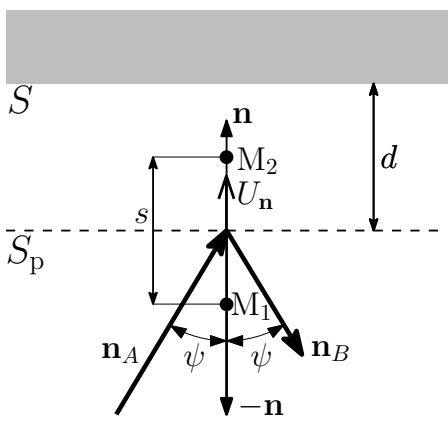

Figure 4. Orientation of the propagation directions of wave $A$ and wave $B$ with respect to the surface normal of surface $S_{\mathrm{p}}$. The positions of the microphones $\mathrm{M}_{1}$ and $\mathrm{M}_{2}$ are depicted by the circular markers.

For a pp-probe with microphones $\mathrm{M}_{1}$ and $\mathrm{M}_{2}$, spaced a distance $s$ apart, see Fig. 4 , we equate the acoustic pressures of the approximated- and the actual field, and obtain

$$
\left[\begin{array}{ll}
e^{i k \frac{s}{2} \cos (\psi)} & e^{-i k \frac{s}{2} \cos (\psi)} \\
e^{-i k \frac{s}{2} \cos (\psi)} & e^{i k \frac{s}{2} \cos (\psi)}
\end{array}\right]\left[\begin{array}{l}
A(\mathbf{r}) \\
B(\mathbf{r})
\end{array}\right]=\left[\begin{array}{l}
P_{1}(\mathbf{r}) \\
P_{2}(\mathbf{r})
\end{array}\right]
$$

where $k$ is the wavenumber, and $P_{1}(\mathbf{r})=P\left(\mathbf{r}-\frac{s}{2} \mathbf{n}\right)$ and $P_{2}=P\left(\mathbf{r}+\frac{s}{2} \mathbf{n}\right)$ are the complex acoustic pressures measured by microphones $\mathrm{M}_{1}$ and $\mathrm{M}_{2}$, respectively. It follows that

$$
\begin{aligned}
& A=\frac{i}{D}\left[P_{2} \exp \left(-i k \frac{s}{2} \cos \psi\right)-P_{1} \exp \left(i k \frac{s}{2} \cos \psi\right)\right], \\
& B=\frac{i}{D}\left[P_{1} \exp \left(-i k \frac{s}{2} \cos \psi\right)-P_{2} \exp \left(i k \frac{s}{2} \cos \psi\right)\right],
\end{aligned}
$$


where $D=2 \sin (k s \cos \psi)$. The solutions for $A(\mathbf{r})$ and $B(\mathbf{r})$ become singular for frequencies

$$
f_{q}=\frac{q c_{0}}{2 s \cos \psi}
$$

where $q=1,2,3, \ldots$. For a microphone spacing $s=20 \mathrm{~mm}$, the lowest frequency $f_{1}$ at which the singularity occurs equals ca. $8600 \mathrm{~Hz}$ for normal incidence. $f_{1}$ increases with increasing angle of incidence. Having determined the complex amplitudes $A(\mathbf{r})$ and $B(\mathbf{r})$ either with a pu- or a pp-probe, the incident acoustic intensity in the direction $\mathbf{n}$ (towards the material surface $S$ ) and the reflected acoustic intensity in the opposite direction, can be calculated according to

$$
\begin{aligned}
I_{\text {in }}(\mathbf{r}) & =\frac{|A(\mathbf{r})|^{2}}{2 \rho_{0} c_{0}} \cos \psi, \\
I_{\text {refl }}(\mathbf{r}) & =\frac{|B(\mathbf{r})|^{2}}{2 \rho_{0} c_{0}} \cos \psi .
\end{aligned}
$$

The active acoustic intensity in direction $\mathbf{n}$ can be calculated with the well-known formula by Fahy [19]:

$$
I_{\mathrm{ac}}(\mathbf{r})=\frac{1}{2} \operatorname{Re}\left[P(\mathbf{r}) \overline{U_{\mathrm{n}}(\mathbf{r})}\right] .
$$

Whereas application of this equation is straightforward for a pu-probe, for a pp-probe it is less obvious. If we would use the finite difference (FD) approximation to determine the particle velocity for use in Eq. (14), see Fahy [19]:

$$
U_{n}(\mathbf{r}) \approx \frac{i}{Z_{0} k s}\left[P_{2}(\mathbf{r})-P_{1}(\mathbf{r})\right],
$$

our formulation would become inconsistent. One can use the FD-approximation in Eq. (15) and then use Eq. (14) to determine the active acoustic intensity, but then one should have used Eq. (6) to determine $A(\mathbf{r})$ using the formulation for a pu-probe to calculate the incident acoustic intensity. However, in that case, both the active- and the incident acoustic intensity will suffer from the finite difference error, known for pp-probes [19].

Instead, we determine the particle velocity at the geometric center of the pp-probe in direction $\mathbf{n}$ using the complex wave amplitudes $A(\mathbf{r})$ and $B(\mathbf{r})$ according to the LSPW assumption:

$$
U_{\mathbf{n}}(\mathbf{r})=\frac{\cos (\psi)}{Z_{0}}[A(\mathbf{r})-B(\mathbf{r})]
$$

so that, with Eq. (14), it follows that the active acoustic intensity equals the difference between the incident- and reflected acoustic intensity:

$$
I_{\mathrm{ac}}(\mathbf{r})=\frac{|A(\mathbf{r})|^{2}-|B(\mathbf{r})|^{2}}{2 Z_{0}} \cos (\psi) .
$$

Having estimated the active- and incident acoustic intensity by the above procedure, the area-averaged oblique sound absorption coefficient can straightforwardly be calculated with Eq. (1) after spatial integration of both intensities according to Eqs. (2) and (3), respectively. It is pointed out that the estimated intensities will be exact if the actual acoustic field matches with the field described by the local specular plane wave assumption.

\section{Implementation}

The measurements presented in this paper were performed using a newly developed 3D pp-sound intensity probe, see Fig. 5. The 8 microphones used in this probe are digital, omnidirectional, MEMS-microphones (ADMP441, Analog Devices, $4.7 \times 3.8 \times 1.0 \mathrm{~mm})$. They have a signal-to-noise ratio of $61 \mathrm{~dB}(\mathrm{~A})$ and a dynamic range of $87 \mathrm{~dB}$. The microphones are spaced $20.0 \mathrm{~mm}$ in the $\mathrm{x}$ - and $\mathrm{y}$-direction, and $23.1 \mathrm{~mm}$ in the z-direction. As reported in an earlier paper [14], some diffraction effects between 4 and $6 \mathrm{kHz}$ were observed during a series of calibration measurements. The resulting phase- and amplitude errors at these frequencies can, particularly for poorly absorbing samples, result in noticeable inaccuracies in the sound absorption curve, as observed in the same paper. However, the samples described in Sec. 4 are good sound absorbers, and the obtained absorption curves do not seem to be influenced by phase- and amplitude errors.

Due to the particular design of the probe, we have chosen an implementation of the LSPW-method that is somewhat different from the theory in the preceding section. The first aspect of this implementation is the application of a least-squares method to obtain the complex amplitudes $A$ and $B$.

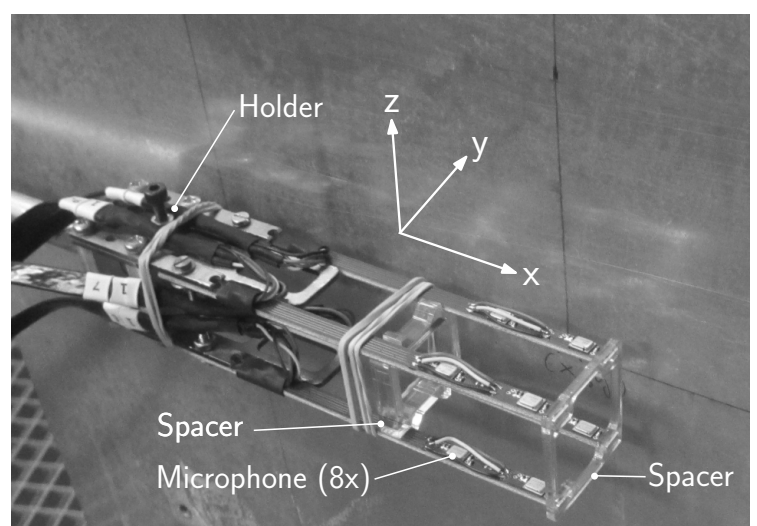

Figure 5. 3D sound intensity probe in front of a plate. The $\mathrm{y}$-direction points normally into this plate. 
we have chosen to solve $A$ and $B$ from the following set of equations instead:

$$
\mathbf{M v}=\mathbf{P}
$$

in which

$$
\mathbf{M}=\left[\begin{array}{cc}
M_{11} & M_{12} \\
\vdots & \vdots \\
M_{N 1} & M_{N 2}
\end{array}\right], \mathbf{v}=\left[\begin{array}{c}
A \\
B
\end{array}\right], P=\left[\begin{array}{c}
P 1 \\
\vdots \\
P_{N}
\end{array}\right]
$$

where $N$ is the number of microphones. The elements of the first and second column of the matrix $\mathbf{M}$ are given by

$$
\begin{aligned}
& M_{j 1}=e^{-i k\left[x_{j} \sin \phi \sin \theta+y_{j} \sin \phi \cos \theta+z_{j} \cos \phi\right]}, \\
& M_{j 2}=e^{-i k\left[x_{j} \sin \phi \sin \theta-y_{j} \sin \phi \cos \theta+z_{j} \cos \phi\right]},
\end{aligned}
$$

where $x_{j}, y_{j}$, and $z_{j}$ are the spatial coordinates of microphone $j$ relative to the geometric center of probe according to Fig. 5. One can solve the overdetermined system of equations in Eq. (18) for the complex amplitudes in $\mathbf{v}$ in a least-squares sense [20] by pre-multiplication of the left- and right-hand side with the Hermitian transpose $\mathbf{M}^{\mathrm{H}}$, and then solving for $\mathbf{v}$, according to:

$$
\mathbf{v}=\left(\mathbf{M}^{\mathrm{H}} \mathbf{M}\right)^{-1} \mathbf{M}^{\mathrm{H}} \mathbf{P}
$$

By doing so, the real-valued, semi-positive definite functional

$$
F=(\mathbf{M v}-\mathbf{P})^{\mathrm{H}}(\mathbf{M v}-\mathbf{P})
$$

being the sum of all squared errors, is minimized. I.e. the above procedure tries to determine $A$ and $B$, such that a least-squares fit to the measured acoustic pressures is obtained. The above solving procedure has to be repeated for every discrete frequency in the complex spectrum $\mathbf{P}$ that falls within the frequency range of interest.

The second aspect of our implementation is an adaption of the system of equations (18). The measurement of the acoustic pressures in Sec. 4 is performed without a reference signal. Therefore, spectral averaging of the complex spectrum of each of the acoustic pressures $P_{1} \ldots P_{N}$ is useless. In such cases, one defines one of the data channels to be the reference signal and calculates the transfer functions or cross-power spectra, which are suitable for spectral averaging. Without loss of generalization, we have defined channel 1 as the reference signal. If cross-power spectra are used, the system of equations (18) can be formulated as

$$
\left[\begin{array}{cc}
M_{11} & M_{12} \\
\vdots & \vdots \\
M_{N 1} & M_{N 2}
\end{array}\right]\left[\begin{array}{c}
A^{\prime} \\
B^{\prime}
\end{array}\right]=\left[\begin{array}{c}
\overline{P_{1}} P 1 \\
\vdots \\
\overline{P_{1}} P_{N}
\end{array}\right]
$$

where $A^{\prime}=\overline{P_{1}} A$ and $B^{\prime}=\overline{P_{1}} B$, the overbar denotes complex conjugation, and the explicit dependence on $\mathbf{r}$ has been omitted. As the solving procedure now yields $A^{\prime}$ and $B^{\prime}$ instead of $A$ and $B$, one has to divide the right-hand side of Eqs. (12), (13) and (17) by the auto-power spectrum of the reference channel $\left|P_{1}\right|^{2}$ to obtain the correct incident, reflected, and active intensity, respectively. Once the active- and incident intensity are known, one can proceed to calculate the area-averaged sound absorption coefficient, as outlined in Sec. 1.

\section{Measurements}

\subsection{Sample 1: sound absorbing foam}

The measurements described in this section were performed for a $50 \mathrm{~mm}$ thick sheet of melamine resin foam, Basotect $\mathrm{G}$, backed by a $10 \mathrm{~mm}$ thick aluminum plate, see Fig. 6. The dimensions of the foam sheet are $1250 \times 625 \mathrm{~mm}$, whereas the dimensions of the aluminum plate are slightly larger. The foam was attached to the plate using double-sided adhesive tape.

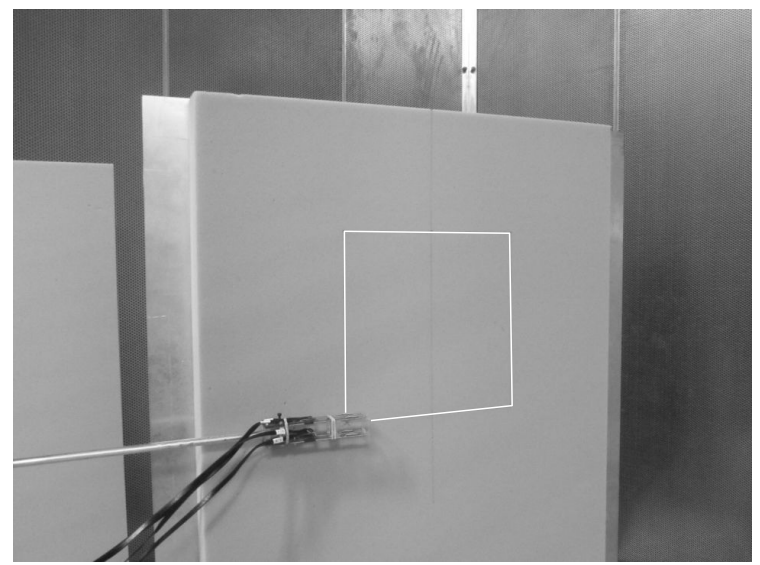

Figure 6. Probe in front of foam sample backed by a thick plate. The scanning area is indicated in white.

The sample was positioned vertically in a wellabsorbing, but not anechoic, room with approximate dimensions $6 \times 6 \times 5 \mathrm{~m}$. The measurement was performed using the 3D sound intensity probe shown in Fig. 5. The probe was moved along the surface of the sample by means of a PC-controlled scanning system, maintaining a distance $d=20 \mathrm{~mm}$ of the geometric center of the probe to the surface of the sample. 
The positioning accuracy of the scanning system is less than $1 \mathrm{~mm}$. A square, equidistant grid of $21 \times 21$ points spaced at $12.8 \mathrm{~mm}$ was defined upon the foam surface, resulting in a measurement surface of $256 \times 256 \mathrm{~mm}^{2}$. At each point, the acoustic pressures were recorded during $10 \mathrm{~s}$. After each moving step of the scanning system, sufficient time was allowed for residual vibration to dampen out before the start of a new recording.

A small loudspeaker, with a membrane diameter of $23 \mathrm{~mm}$ and a circular housing having a diameter of $80 \mathrm{~mm}$, served as a sound source. It was positioned at $1 \mathrm{~m}$ from the plate such that the desired angle of incidence occurs at the geometric center of the measurement region. Temperature, ambient pressure and relative humidity were recorded at the start of the scanning session. Data acquisition is performed using a 40-channel digital front-end and a PC. The signals were processed in data blocks of 4096 points, with $80 \%$ overlap. The frequency resolution $\Delta f$ of all results shown hereafter, equals $11.7 \mathrm{~Hz}$. The lower frequency limit of the results is set to $1300 \mathrm{~Hz}$ as the small speaker did not radiate sufficient acoustic power to maintain a coherence between the microphone signals above 0.9 .

Two measurements were carried out: a normal incidence and a $45^{\circ}$ incidence measurement. To verify the results, the Delany-Bazley model [21] is used. First, the model is fitted to normal incidence sound absorption curve by varying the flow resistivity. Then, the normal specific acoustic surface impedance of the rigidly backed foam layer is calculated with the model. Having obtained this impedance, we calculated two predictions of the sound absorption curve for the second measurement, i.e. for $45^{\circ}$ incidence. One prediction assumes local reaction, and the second prediction assumes extended reaction. Both predicted curves are compared with the curve for $45^{\circ}$ incidence obtained with the LSPW-method.

In the first, normal incidence measurement, the effective area-averaged sound absorption coefficient was determined with the LSPW-method while accounting for the variation of the angle of incidence with position. The maximum angle of incidence equals $10^{\circ}$, occurring at the corners of the square measurement grid. A separate analysis for subsets of points having the same angle of incidence, showed that the variation of the area-averaged sound absorption coefficient with incidence angle was not noticeable, as expected. Therefore, the effective area-averaged sound absorption coefficient can be interpreted as the result of a purely normal incidence measurement.

Fig. 7 shows the sound absorption curve obtained with the LSPW-method, a theoretical curve based on

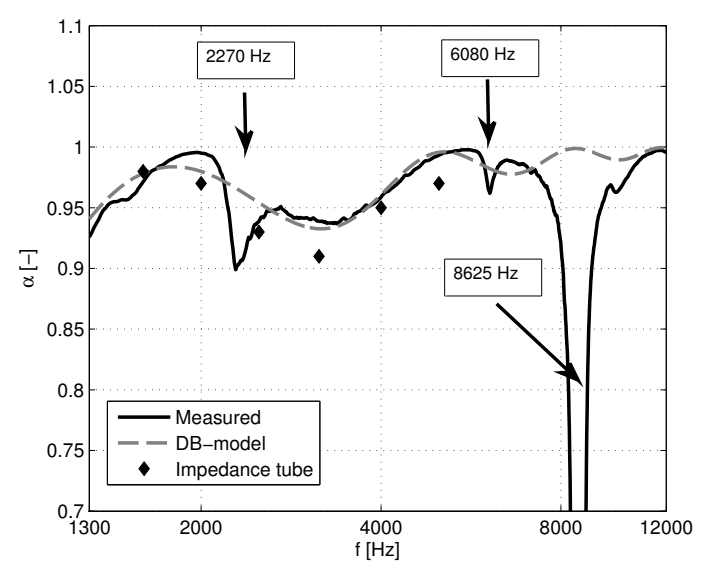

Figure 7. Normal incidence sound absorption coefficient vs. frequency, measured with the LSPW-method, calculated with the model of Delany and Bazley (DB-model), and measured with an impedance tube acc. ISO 105342 [22] for a layer thickness of $51 \mathrm{~mm}$ (manufacturer data, $1 / 3$-octave values).

the model by Delany and Bazley [21], and impedance tube-based data from the manufacturer of the foam. The theoretical curve was determined by fitting the Delany-Bazley model to the measurement results by varying the flow resistivity, being the case for a flow resistivity $\sigma$ of $8000 \mathrm{Ns} / \mathrm{m}^{4}$. This value is realistic as Garai and Pompoli [23] reported an average of ca. $\sigma=10000 \mathrm{Ns} / \mathrm{m}^{4}$ from an inter-laboratory test (round-robin) for a Basotect foam with a mass density of $10 \pm 1.5 \mathrm{~kg} / \mathrm{m}^{3}$, whereas the Basotect $\mathrm{G}$ foam in our measurements is specified to have a mass density of $9+2 /-1 \mathrm{~kg} / \mathrm{m}^{3}$. Furthermore Kino et al. [24] reported $\sigma=6197 \mathrm{Ns} / \mathrm{m}^{4}$ for Basotect TG with a mass density of $8.77 \mathrm{~kg} / \mathrm{m}^{3}$. Please note that we have extrapolated the theoretical curve obtained with the Delany-Bazley model up to 12 $\mathrm{kHz}$, whereas Delany and Bazley indicated validity of their model up to $f / \sigma=1$, being $8 \mathrm{kHz}$ in our case.

The black solid curve in Fig. 7 is obtained with the LSPW-method. The distinct dips in this curve at 2273 and $6080 \mathrm{~Hz}$ are related to a variation of the local sound absorption coefficient within a part of the area of the measurement surface. This variation is caused by local detachment of the foam from the adhesive tape, and was analyzed more in detail by the authors in [14].

The Delany-Bazley model predicts the measured curve very well, and agreement with the impedance tube results is quite good. The strong dip at $8625 \mathrm{~Hz}$ in the measured curve is caused by the singularity of $A$ and $B$ in Eqs. (9) and (10). At this frequency, one-half wavelength equals the microphone spacing in the surface normal direction, i.e. the $y$-direction in Fig. 5. 
The Delany-Bazley model will now be used to predict the sound absorption curve for the second measurement having $45^{\circ}$ incidence. To this purpose, the specific normal acoustic surface impedance for the rigidly-backed foam layer is calculated first, using

$$
Z_{\mathrm{S}}=-i Z_{\mathrm{c}} \cot \left(k_{\mathrm{c}} t\right)
$$

where $t$ is the thickness of the layer of foam. $Z_{\mathrm{c}}$ and $k_{\mathrm{c}}$ are the characteristic specific acoustic impedance and complex wavenumber of the foam obtained from the Delany-Bazley model, respectively. We will now use the calculated specific normal acoustic surface impedance obtained with Eq. (24) to predict the sound absorption coefficient for $45^{\circ}$ incidence. This prediction allows us to verify the sound absorption coefficient measured with the LSPW-method for that angle in the second measurement. Two predictions, $\alpha_{\mathrm{LR}}$ and $\alpha_{\mathrm{ER}}$, are calculated, where the subscripts indicate locally- or extended reaction. The first prediction assumes a locally reacting surface, so that the following equation can be used to calculate the sound absorption coefficient:

$$
\alpha_{\mathrm{LR}}(\psi)=\frac{4 \operatorname{Re}\left(Z_{\mathrm{S}}^{\prime}\right) \cos \psi}{\left[1+\operatorname{Re}\left(Z_{\mathrm{S}}^{\prime}\right) \cos \psi\right]^{2}+\left[\operatorname{Im}\left(Z_{\mathrm{S}}^{\prime}\right) \cos \psi\right]^{2}}
$$

where $Z_{\mathrm{S}}^{\prime}=Z_{\mathrm{S}} / Z_{0}$. The second prediction is performed for an extended reaction surface and hence, we first have to calculate the normal specific acoustic surface impedance for oblique incidence. To this purpose we use Eq. (8) in Davies and Mulholland [3] for an isotropic rigidly-backed absorbing layer, originating from Brekshovskikh [25]:

$$
\begin{aligned}
Z_{\mathrm{S}}^{\prime}(\psi)= & \frac{Z_{\mathrm{c}}}{Z_{0}} \frac{i}{\left[1-\left(\frac{k}{k_{\mathrm{c}}} \sin \psi\right)^{2}\right]} \times \\
& \cot \left[k_{\mathrm{c}} t\left(1-\frac{k}{k_{\mathrm{c}}} \sin \psi\right)^{1 / 2}\right]
\end{aligned}
$$

The second prediction, $\alpha_{\mathrm{ER}}(\psi)$, is then calculated by substituting $Z_{\mathrm{S}}^{\prime}(\psi)$ obtained with Eq. (26) in Eq. (25)

Both predictions, and the curve for $45^{\circ}$ incidence determined with the LSPW-method are shown in Fig. 8. The measurement result indicates that the melamine resin foam is rather of the extended reaction type than of the locally reacting type, which is plausible as its flow resistivity is low. The measured curve agrees quite well with the extended reaction prediction. Although the qualitative course of the predicted and measured curve match very well, the frequencies at which maximum absorption occurs, do not coincide. This could indicate that the

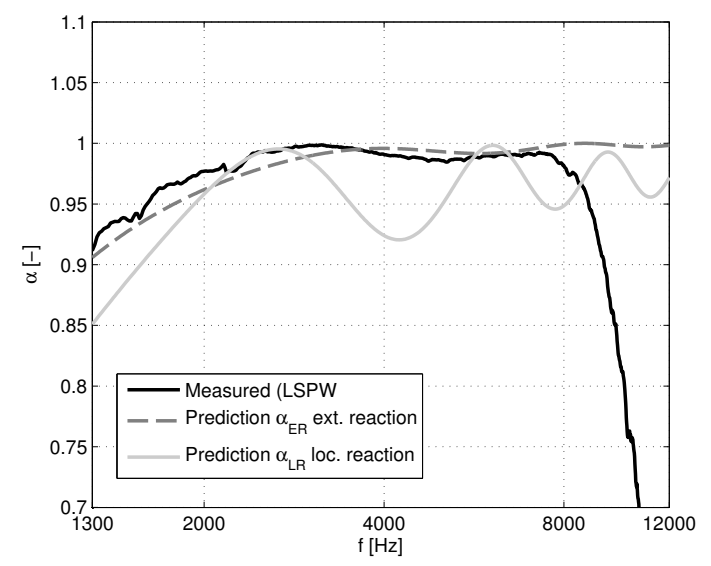

Figure 8. Oblique incidence sound absorption coefficient for $45^{\circ}$ incidence vs. frequency, measured with the LSPWmethod, predicted for a locally-reacting surface $\alpha_{\mathrm{LR}}$, and predicted for an extended reaction surface $\alpha_{\mathrm{ER}}$.

angle of refraction inside the material is smaller in reality than predicted by the extended reaction model

The measured (black) curve rapidly already starts to deviate from its expected course above $8 \mathrm{kHz}$. However the singularity in the complex amplitudes $A$ and $B$ occurs only at $12.2 \mathrm{kHz}$. The cause of the deviation lies in the presence of phase errors of the probe. The sensitivity to such errors increases with an increasing angle of incidence. To obtain accurate results at large angles of incidence, a microphone spacing larger than $20 \mathrm{~mm}$ is necessary given the phase errors of the probe. However, a larger spacing causes the frequency at which singularity occurs to decrease, so that accurate measurements that involve combinations of large angles of incidence and high frequencies are difficult to realize.

Besides the purpose of the prediction of the sound absorption curve for the second measurement, we can also use the calculated specific normal acoustic surface impedance according Eq. (24) to demonstrate that area-averaging is also useful when measuring the specific normal acoustic surface impedances in non-ideal fields. To this purpose, the complex wave amplitudes $A$ and $B$, obtained with the LSPWmethod, are used to determine the specific normal acoustic surface impedance of the rigidly-backed layer of foam.

First, the local normal specific acoustic surface impedance at the geometric center of the probe is determined. Using the LSPW assumption, this impedance can be expressed in terms of $A^{\prime}$ and $B^{\prime}$, as obtained by solving the system of equations in Eq. (23):

$$
Z_{\mathrm{M}}=\frac{Z_{0}}{\cos (\psi)} \frac{A^{\prime}+B^{\prime}}{A^{\prime}-B^{\prime}}
$$


Assuming plane wave propagation between the measurement surface and the material surface, the specific normal acoustic surface impedance can be calculated from $Z_{\mathrm{M}}$, using Eq. (4) in [4] by Allard et al.:

$$
Z_{\mathrm{S}}(\psi)=\frac{Z_{0}}{\cos \psi}\left[\frac{Z_{\mathrm{M}} \cos \psi-i Z_{0} \tan (k d \cos \psi)}{Z_{0}-i Z_{\mathrm{M}} \cos \psi \tan (k d \cos \psi)}\right]
$$

Figure 7 shows the specific normal acoustic surface impedance as calculated with the Delany-Bazley model, using Eq. (24), and as determined from the measurements using Eq. (28). The gray bands indicate the envelope that contains the curves for all 441 measurement points. The agreement of the mean value of these sets of curves (solid black line) with the calculated curve (dashed line) is very good, for both the real and imaginary part. Hence, we conclude that area-averaging is also effective in measuring the normal acoustic surface impedance in non-ideal fields.
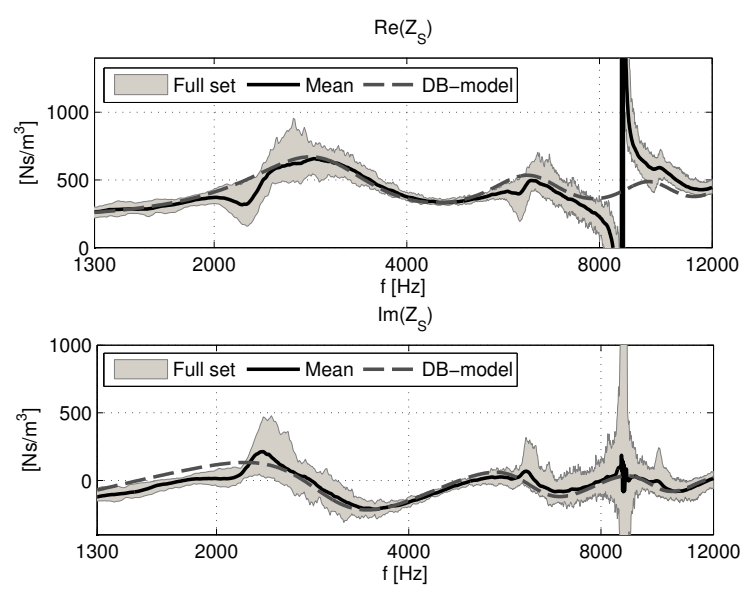

Figure 9. Normal specific acoustic surface impedance, measured with the LSPW-method and calculated with the model of Delany and Bazley. Upper graph: real part. Lower graph: imaginary part.

\subsection{Sample 2: Periodic absorber}

The second sample is a periodic absorber, see Fig. 10. This type of absorber was chosen to investigate how the LSPW-method deals with acoustic fields that locally differ strongly from the field assumed by the LSPW assumption. This is the case for the periodic absorber investigated here. Periodic absorbers are characterized by discrete frequencies at which scattering of so-called radiating harmonics at non-specular angles is cut-on. At- and beyond the first cut-on frequency, the acoustic field consists of an incident wave, a specularly reflected wave, and one or multiple scattered waves. In addition, evanescent surface waves are present in the near-field.
The dimensions of the sample are $120 \times 120 \mathrm{~cm}$. The wooden ribs are $50 \mathrm{~mm}$ wide and are spaced $50 \mathrm{~mm}$ apart. The thickness of the wooden ribs varies between 46.5 and $48.5 \mathrm{~mm}$. The melamine resin foam that has been analyzed in the preceding section, is used to fill the slots between the ribs, where a thickness of $47.5 \mathrm{~mm}$ was chosen. The backing of the whole plate can be considered to be rigid. It consists of 2 layers of a $12 \mathrm{~mm}$ thick MDF-board (medium density fiber board).

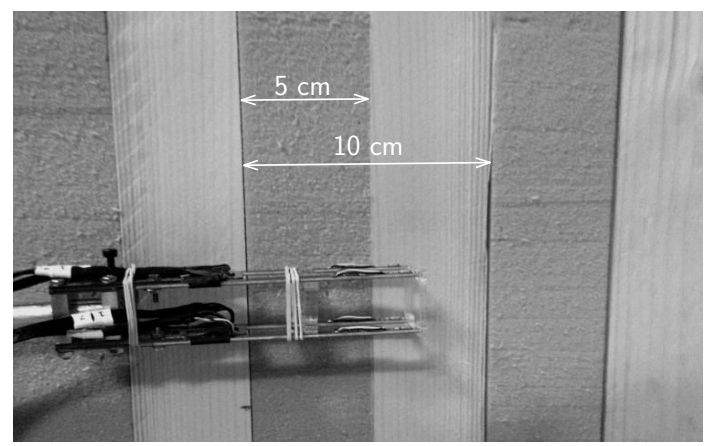

Figure 10. Probe in front of periodic absorber.

Two measurements were performed: a normal incidence and a $30^{\circ}$ incidence measurement. A square scanning area, centered on the sample's surface, with a width of $0.2 \mathrm{~m}$ ( 2 spatial periods) was defined. All other parameters of the setup are identical to the setup described in Sec. 4.1. In this section, results are presented up to $7000 \mathrm{~Hz}$ as there is no point in extending this frequency for the phenomena that we are discussing in this section. The predictions are based again on the model by Delany and Bazley [21] where $\sigma=8000 \mathrm{Ns} / \mathrm{m}^{4}$.

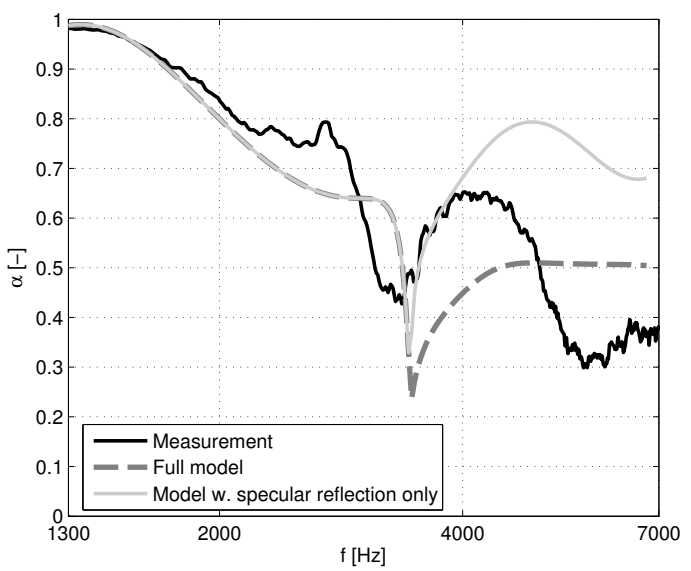

Figure 11. Normal incidence sound absorption coefficient vs. frequency, measured with the LSPW-method, predicted using a full model including scattering, and predicted using a model that only includes the specularly reflected wave. 
Fig. 11 shows the area-averaged normal and incidence sound absorption coefficient along with two theoretical predictions. Both predictions were calculated using the approach by Mechel [26] for a semi-free field bounded by a periodic absorber that is subjected to plane wave incidence. The first prediction, indicated by the dashed gray curve, accounts for scattered waves and includes radiating harmonics up to $4^{\text {th }}$ order. The second prediction only includes the first order radiating harmonic and thus only accounts for specular reflection. It is represented by the solid, light gray curve.

Up to $2 \mathrm{kHz}$ is the agreement is very good. Beyond this frequency, the measured curve deviates from the predicted curves. This deviation is almost certainly caused by the presence of evanescent surface waves that exist for every order of the radiating harmonics below their cut-on frequency. Consequently, the estimates for both the activeand incident acoustic intensity will be more or less inaccurate as the probe is located in the near-field. This field is very different from the set of two waves assumed by the local specular plane wave assumption.

At $3450 \mathrm{~Hz}$, oblique scattering into the far-field occurs. The LSPW-method is quite accurate at this frequency although the acoustic field differs much from the local specular wave assumption. Even in the presence of two obliquely scattered waves, the LSPW-method seems to be capable of determining the amplitude of the specularly reflected wave as the measured curve agrees well with the specular wavebased prediction up to $3900 \mathrm{~Hz}$. It is pointed out that the amplitude of the next order evanescent surface waves is still relatively low in this frequency range, but steadily increases with increasing frequency up to the frequency at which a new radiating harmonic is cut on.

Figure 12 shows the same curves for $30^{\circ}$ incidence. The observations stated for normal incidence up to the cut-on frequency, $2300 \mathrm{~Hz}$, of the first scattered wave also seem to be valid here. Above this frequency, however, the measured curve agrees less good with the specular reflection-based prediction (light-gray curve) than for the normal incidence case. This can be explained by the relatively higher amplitude of the evanescent surface waves compared to the normal incidence case, as verified with the model. To obtain an accurate estimate for the sound absorption coefficient, the complex wave amplitudes $A$ and $B$ must be determined in the far-field.

From the results, we conclude that the LSPWmethod works well as long as scattering does not occur and as long as the measurement is not influenced by evanescent surface waves. To be able to measure the sound absorption coefficient of the

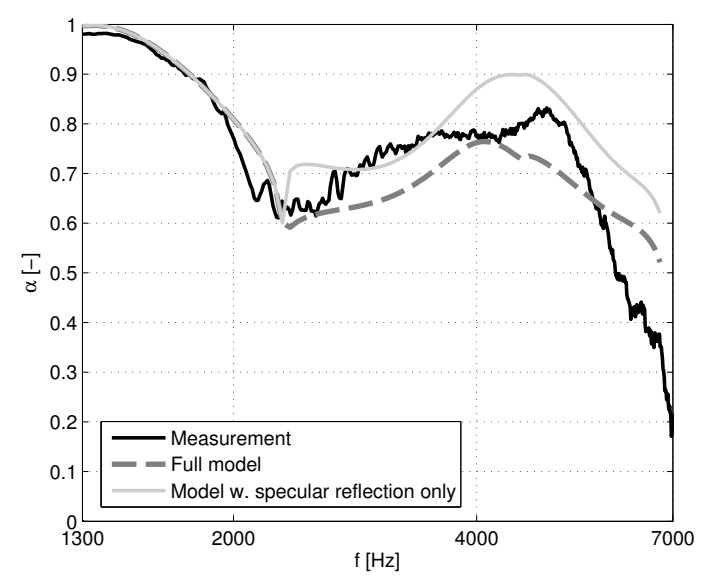

Figure 12. Sound absorption coefficient vs. frequency for $30^{\circ}$ incidence, measured with the LSPW-method, predicted using a full model including scattering, and predicted using a model that only includes the specularly reflected wave.

periodic absorber up the first cut-on frequency, the measurement has to be performed at a greater distance $d$ from the sample's surface. Furthermore, the results seem to indicate that the LSPW-method is also capable of determining the amplitude of the specularly reflected wave above the first cut-on frequency, provided that the measurement is not influenced by evanescent surface waves.

\section{Conclusions}

In this paper the LSPW-method for the measurement of the area-averaged oblique incidence sound absorption coefficient is presented. Its underlying assumption assumes that, locally, the acoustic field can be approximated by a set of two plane waves that represent local specular reflection. There is no need for an overall model of the acoustic field in which one has to account for the physical behavior of the sample and/or for the directivity characteristics of the source.

To ensure that the angle of incidence is about constant over the measurement surface, the dimensions of the measurement surface must be significantly smaller than the source distance. If this is not the case, one obtains an effective sound absorption coefficient. The LSPW-method does not account for geometrical spreading of the waves between the measurement surface and the surface of the sample, so that a large source distance is also helpful in reducing errors that may be caused by neglecting geometrical spreading.

Measurements performed for a foam sample subjected to normal and $45^{\circ}$ incidence, show that the LSPW-method yields accurate results, although these measurements were performed in a non-ideal acoustic field. Effects that typically negatively influence the 
results of single point-based measurements, such as the presence of room reflections, are effectively reduced by employing area-averaging. It is also shown that the LSPW-method can be used to determine the specific normal acoustic surface impedance, and that area-averaging is also useful for this purpose. Measurements for a periodic absorber indicate that the LSPW-method can be used up to the cut-on frequency of scattering, provided that the measurement is not performed in the near-field of the absorber due to the presence of evanescent surface waves.

We conclude that the LSPW-method is a useful alternative for measuring the oblique incidence sound absorption coefficient while avoiding the effort of generating an overall model of the acoustic field.

Future work may include investigations for large angles of incidence and investigations of the effects of phase- and amplitude errors of the probe.

\section{Acknowledgements}

The authors would like to thank CAE-Systems, Gütersloh, Germany, for supplying the prints with the MEMS-microphones. In addition, the support of the Engineering Fluid Dynamics group at the University of Twente during the measurements is gratefully acknowledged.

\section{References}

[1] M. Vorländer: Computer simulations in room acoustics : Concepts. J. Acoust. Soc. Am. 133 (2013) 12031213.

[2] ISO 354:2003: Acoustics - Measurement of sound absorption in a reverberation room. 2003.

[3] J. Davies, K. Mulholland: An impulse method of measuring normal impedance at oblique incidence. J. Sound Vib. 67 (1979) 135-149.

[4] J. Allard, R. Bourdier, A. Bruneau: The measurement of acoustic impedance at oblique incidence with two microphones. J. Sound Vib. 101 (1985) 130-132.

[5] M. Garai: Measurement of the sound-absorption coefficient in situ: The reflection method using periodic pseudo-random sequences of maximum length. Appl. Acoust. 39 (1993) 119-139.

[6] E. Mommertz: Angle-dependent in-situ measurements of reflection coefficients using a subtraction technique. Appl. Acoust. 46 (1995) 251-263.

[7] C. Nocke: In-situ acoustic impedance measurement using a free-field transfer function method. Appl. Acoust. 59 (2000) 253-264.

[8] R. Lanoye, G. Vermeir, W. Lauriks, R. Kruse, V. Mellert: Measuring the free field acoustic impedance and absorption coefficient of sound absorbing materials with a combined particle velocity-pressure sensor. J. Acoust. Soc. Am. 119 (2006) 2826-2831.

[9] R. Kruse: Application of the two-microphone method for in-situ ground impedance measurements. Acta Acust. Acust. 93 (2007) $837-842$.
[10] E. Tijs, E. Druyvesteyn: An intensity method for measuring absorption properties in situ. Acta Acust. Acust. 98 (2012) 342-353.

[11] S. Liu, K. M. Li: Efficient computation of the sound fields above a layered porous ground. The Journal of the Acoustical Society of America 131 (2012) 43894398.

[12] K. M. Li, S. Liu: Propagation of sound from a monopole source above an impedance-backed porous layer. The Journal of the Acoustical Society of America 131 (2012) 4376-4388.

[13] E. Kuipers, Y. Wijnant, A. de Boer: A numerical study of a method for measuring the effective in situ sound absorption coefficient. J. Acoust. Soc. Am. 132 (2012) EL236-42.

[14] E. Kuipers, Y. Wijnant, A. de Boer: Measuring sound absorption: considerations on the measurement of the active acoustic power (submitted). Acta Acust. Acust. (2013).

[15] Y. Wijnant, E. Kuipers, A. de Boer: Development and application of a new method for the in-situ measurement of sound absorption. ISMA 31, Leuven, Belgium, 2010.

[16] E. Kuipers, Y. Wijnant, A. de Boer: Theory and application of a new method for the in-situ measurement of sound absorption. DAGA 2011, Düsseldorf, 2011, DEGA, Berlin, 723-724.

[17] E. Kuipers, Y. Wijnant, A. de Boer: In situ sound absorption measurement: investigations on oblique incidence. DAGA 2012, Darmstadt, 2012, DEGA, Berlin, 351-352.

[18] H.-E. de Bree: An overview of Microflown technologies. Acta Acust. Acust. 89 (2003) 163-172.

[19] F. Fahy: Sound intensity. 2nd ed. E \& FN Spon, London, 1995.

[20] A. Björck: Numerical methods for least squares problems. SIAM, 1996.

[21] M. E. Delany, E. N. Bazley: Acoustical properties of fibrous absorbent materials. Appl. Acoust. 3 (1970) $105-116$.

[22] ISO 10534-2:1998: Acoustics - Determination of sound absorption coefficient and impedance in impedance tubes - Part 2: Transfer-function method. 1998.

[23] M. Garai, F. Pompoli: A European inter-laboratory test of airflow resistivity measurements. Acta Acust. Acust. 89 (2003) 471-478.

[24] N. Kino, T. Ueno, Y. Suzuki, H. Makino: Investigation of non-acoustical parameters of compressed melamine foam materials. Applied Acoustics $\mathbf{7 0}$ (2009) 595-604.

[25] L. Brekhovskikh: Waves in layered media. Academic Press, New York, 1960.

[26] F. Mechel: Sound fields at periodic absorbers. J. Sound Vib. 136 (Jan. 1990) 379-412. 